\title{
010 PP EXPLORING THE IMPACT OF A CANCER AWARENESS TRAINING PROGRAMME: A QUALITATIVE STUDY OF COMMUNITY-BASED HEALTH STAFF AND VOLUNTEERS
}

K Osborne, ${ }^{*}$ E Power. Cancer Research UK, UK *Presenting author.

10.1136/bmjopen-2015-UCLSymposiumAbstracts.21

Background Cancer Research UK's cancer awareness training programme, Talk Cancer, aims to equip and empower frontline healthcare staff and volunteers to raise awareness of cancer and promote health behaviour change in their community. Each workshop aims to increase knowledge of cancer prevention, screening and early diagnosis and improve confidence communicating with the public about these areas. This study evaluated the impact of workshops conducted across England between April 2013 and March 2014.

Methods Fourteen in-depth qualitative telephone interviews were conducted with attendees approximately four months after training. Interview transcripts were thematically analysed and themes independently verified by two researchers.

Findings After training, attendees reported increased awareness of the importance of early diagnosis and the link between lifestyle behaviours and cancer risk. They reported feeling more open towards cancer and that cancer had become a legitimate topic of conversation. They reported feeling more confident to have conversations about cancer and, for some, this was related to feeling more credible. As a result, attendees felt more able to manage and initiate conversations about cancer. Specifically, many attendees had promoted the importance of early diagnosis and encouraged people to seek help with an unusual or persistent change to their body. Some attendees also said they had visited the GP and made healthy lifestyle changes themselves.

Conclusions Talk Cancer training increased attendees' cancer awareness, openness towards cancer and confidence in having cancer-related conversations. In particular, attendees became advocates for prompt help-seeking after learning about the importance of early diagnosis. Furthermore, reports of attendees making changes to their own behaviour suggest the training may benefit attendees as well as their communities. 